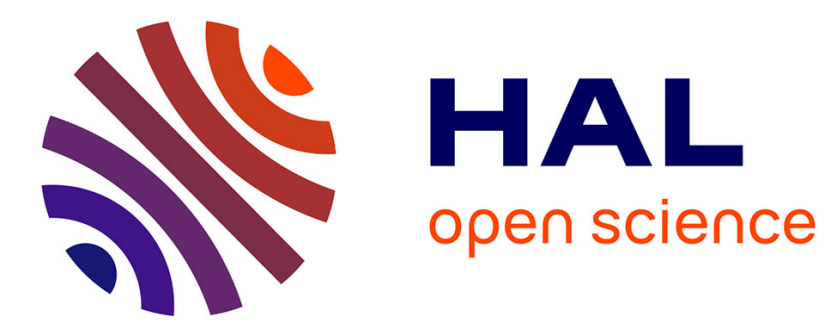

\title{
Automatic Parameter Selection of Image Reconstruction Algorithms for Planar Array Capacitive Imaging
} Carl Tholin-Chittenden, Juan F P J Abascal, Manuchehr Soleimani

\section{To cite this version:}

Carl Tholin-Chittenden, Juan F P J Abascal, Manuchehr Soleimani. Automatic Parameter Selection of Image Reconstruction Algorithms for Planar Array Capacitive Imaging. IEEE Sensors Journal, 2018, 18 (15), pp.6263-6272. 10.1109/JSEN.2018.2844549 . hal-01814533

\section{HAL Id: hal-01814533 \\ https://hal.science/hal-01814533}

Submitted on 13 Jun 2018

HAL is a multi-disciplinary open access archive for the deposit and dissemination of scientific research documents, whether they are published or not. The documents may come from teaching and research institutions in France or abroad, or from public or private research centers.
L'archive ouverte pluridisciplinaire HAL, est destinée au dépôt et à la diffusion de documents scientifiques de niveau recherche, publiés ou non, émanant des établissements d'enseignement et de recherche français ou étrangers, des laboratoires publics ou privés. 


\title{
Automatic Parameter Selection of Image Reconstruction Algorithms for Planar Array Capacitive Imaging
}

\author{
Carl Tholin-Chittenden, Juan Felipe Perez-Juste Abascal and Manuchehr Soleimani
}

\begin{abstract}
Landmines are often be made out of plastic with almost no metallic components which makes detection difficult. A plausible solution is to detect superficial buried plastic objects using planar array Electrical Capacitance Tomography (ECT). Distance detection is a big limiting factor of planar array ECT. Given the ill-posedness and loss of sensitivity with depth, regularization and optimal selection of reconstruction parameters are required for detection. In this work we propose an 'Automatic Parameter Selection' (APS) method for image reconstruction algorithms that selects optimal parameters based on the input data based on a 3 step process. The aim of the first 2 steps is to provide an approximate estimate of the parameters so that future reconstructions can be performed quickly in step 3. To optimise the reconstruction parameters the APS method uses the following metrics. Front Surface Distance Detection (FSDD) is a method of determining an accurate distance measurement from sensor head to object surface in low resolution image reconstructions using interpolation between voxels and Otsu thresholding. CrossSection Reconstruction Score (CSRS) is a simple binary image comparison method which calculates a ratio of expected image to reconstructed image. An initial set of capacitance data was taken for an object at various distances and used to train the APS method by finding the best reconstruction parameters for each distance. Then another set of capacitance data was taken for a new object at different distances than before and reconstructed using the parameters selected by the APS method. The results of this showed that the APS method was able to select unique parameters for each reconstruction which produced accurate FSDDs and consistent CSRSs. This has taken away the need for an expert to manually select parameters for each reconstruction and sped up the process of reconstructions after training. The introduction of FSDD and CSRS is useful as they accurately describe how reconstructions were score and will allow future work to compare results effectively.
\end{abstract}

Index Terms-Electrical Capacitance Tomography Reconstruction Landmine Total-Variation

\section{INTRODUCTION}

Electrical Capacitance Tomography (ECT) is a non-invasive and non-destructive method of imaging through a material under test (MUT) [1]. By measuring the capacitance through the MUT, it is able to build up a permittivity distribution indicating areas of low or high permittivity comparatively. The process requires capacitance data acquisition, environment

Engineering Tomography Lab (ETL), University of Bath, Department of Electronics \& Electrical Engineering, Bath BA2 7AY, United Kingdom (C.Tholin-Chittenden@bath.ac.uk, M.Soleimani@bath.ac.uk)

Univ Lyon, INSA Lyon, Universite Claude Bernard Lyon 1, UJMSaint Etienne, CNRS, Inserm, CREATIS UMR 5220, U1206, Lyon, France (Juan.Abascal@creatis.insa-lyon.fr) modelling, simulation and image reconstruction. ECT systems are often 2-dimensional circular array devices [2] as they are imaging a region which can be accessed from all sides. However when only 1 side is available, the sensor must be flattened into a co-planar array [3]. The modelling must be done in 3 dimensions as capacitance is arching through a 3D space between the sensor electrodes. Due to their orientation the inter-electrode capacitance is reduced thus decreasing detectable distance from the sensor. Previous work on planar array ECT has been able to detect objects as far away from the sensor head as roughly the distance between the furthest apart electrodes [4] which for a $200 \mathrm{~mm}$ square grid-like sensor head was approximately $100 \mathrm{~mm}$ [5]. However the work was vague on how distance is quantified from results and how image reconstruction quality is scored which makes performance comparisons difficult.

One possible application of planar array ECT is landmine detection in which the buried landmine is only detectable from above the ground and sensors cannot be placed all around the viewing region. Current landmine technologies, such as metal detectors, struggle to detect modern landmine technology as they contain almost no metal at all [6]. Theoretically, ECT would be able to detect any material which differed from the ground it was buried in as well as reconstructing a permittivity distribution of the different materials contained in the landmine. This makes it an attractive prospect for future landmine detection technology but in order to get it to a stage where it could realistically be used it needs to be improved in a number of ways. One of these is improving the depth detection range which is currently a big limiting factor of ECT [7]. In order to locate the landmine it is important to know the depth of the landmine and the shape of the landmine which could then also allow identification to be possible. These metrics need to be clearly defined and quantified.

A common method of image reconstruction in ECT is Tikhonov regularisation but Total Variation (TV) algorithms have also received a great deal of attention and many implementations are considered state of the art [8] [9] for image reconstruction. These algorithms are used in many different applications such as medical [10] [11] and industrial [12] [13] with various implementations. Among the available methods for TV minimization, splitting methods, such as proximal algorithms [14] and the split Bregman method [15], [16], are efficient and widely used. However there is no clear winner as each application requires a different reconstruction approach 
with very different parameter selections.

Image reconstruction in an ill-posed problem requires optimal selection of image reconstruction parameters. For L2regularisation, the L-curve or the generalised cross validation are widely used for selecting the regularization parameter [17], [18]. Splitting algorithms have few parameters that need to be tuned. For the case of split Bregman algorithm, previous work provided some guidelines for manual parameter selection [15] [19] [20] as well for automatic selection [21] for some of these parameters. Tikhonov Regularisation has also received attention on parameter selection, both automatic [22] and manual, such as L-Curve method [23].

The aim of this paper is to propose a method of selecting parameters of the image reconstruction algorithms automatically and to assess it on planar array ECT data. The proposed method is applied to a 'Hybrid Tiknovov' with NOSER regularisation [24] and split Bregman based TV methods [15]. The algorithm proposed here will attempt to automate selection of multiple parameters.

Manual reconstruction parameters selection involves an expert who knows how each parameter affects the reconstruction. This is often done through a method of trial and improvement which relies on looking at the result and changing the parameters accordingly. This is usually very time consuming and requires the expert knowledge on the algorithm and application. However a method of automatic parameter selection, which can select reconstruction parameters based only on the measured data, would remove the need for the expert and thus potentially speed up reconstructions and add consistency to results. The proposed method of automatic parameter selection involves finding a set of optimal parameters using a Genetic Algorithm (GA). GAs have been successfully used for parameter tuning in several applications [25] [26].

Previous work in electrical tomography which is investigating distance detection is often vague on quantifying how a distance was calculated [27]. New methods of distance detection and inclusion scoring from 3D reconstructions is proposed.

\section{Electrical CAPACitance TOMOgRAPHY}

ECT is the method of reconstructing a permittivity distribution from a set of capacitance measurements taken between different combinations of electrode pairs. The raw capacitance data measured can be used to infer something about the viewing region in front of the sensor head.

\section{A. Forward Problem}

Before image reconstruction can be performed some precalculations need to be made about the sensor head and the viewing region. This is often referred to as the forward problem and consists of modelling of the environment to understand how a change of permittivity in the viewing region will affect the capacitance measured by the electrodes on the sensor head. To model the relationship between electric potential from the electrodes and the permittivity of the viewing region a combination of Gauss's law is used with Poisson's equation and as there is no charge escaping the viewing region:

$$
\nabla \cdot(\varepsilon \nabla \phi)=0
$$

where $\varepsilon$ is the permittivity distribution, $\phi$ is the electric potential distribution and $\nabla$. is the divergence operator. This is the Partial Differential Equation (PDE) that can be used to calculate electric potential distribution with a fixed permittivity of the viewing area. Capacitance is proportional to the charge on a surface so this can be calculated for one electrode on the sensor head by finding the electric flux density over the surface area of the electrode which can be found by taking the surface integral:

$$
C=\frac{1}{V} \oint_{\Omega} \varepsilon_{0}(x, y, z) \mathbf{E}(x, y, z) d \omega
$$

where $C$ is the inter electrode capacitance, $V$ is the electric potential on the excitation electrode, $\Omega$ is the surface area and $\mathbf{E}$ is the electric field distribution. From this it can be seen that capacitance is a non-linear function of permittivity which can be linearised as follows:

$$
\Delta C=J \Delta \varepsilon
$$

where $J$, the Jacobian, is essentially a conversion matrix that maps a change in permittivity into a change in capacitance values. The number of voxels in the viewing region is defined as $n_{v}$ and the number of unique capacitance measurements, $m$, is dictated by the following equation:

$$
m=\frac{N_{v}\left(N_{v}-1\right)}{2}
$$

where $N_{v}$ is the number of electrodes. This makes the Jacobian size equal to $m \times n_{v}$. Most commonly $n>>m$ depending on the image resolution selected which defines the number of nodes.

\section{B. Inverse Problem}

Equation 3 shows that a permittivity is linearly related to a set of capacitance values. This is a linear ill posed problem, so regularization is needed to obtain a unique solution. Tikhonov regularisation [28] is the most common and simple choice of regularization.

Improvement on standard Tikhonov Regularisation can be achieved by adding NOSER regularisation [24]. Tikhonov regularisation performs very well with ill-posed problems by reducing noise but it tends to over-smooth the output image as it assumes the solution to be smooth. The NOSER method 
is very good at position reconstruction but performs worse with noise which affects the solution more when the object is at larger distances. However the combination of Tikhonov and NOSER perform well. This leads to a 'Hybrid Tikhonov' which is defined by the following equation:

$$
\Delta \varepsilon=\left(J^{T} J+\alpha_{1} \Gamma+\alpha_{2} \operatorname{diag}\left(J^{T} J\right)\right)^{-1} J^{T} \Delta C
$$

where $\Gamma$, the regularisation matrix, is the identity and $\alpha_{1}$ and $\alpha_{2}$ are the scaling parameters of Tikhonov and NOSER respectively. The problem with this solution however is that boundaries gradients between different permittivity levels will be smooth and so they won't show sharply contrasting boundaries. A buried foreign object has very distinct boundaries with the ground around it. For this reason L-2 regularization is not an ideal prior for object detection.

\section{TOTAL VARIATION ALgORITHM}

Total variation methods have been shown to outerperform L2regularization methods in terms of image quality, as TV functional achieves piecewise-constant reconstructions with sharp edges [29]. This is a reasonable assumption in ECT where target objects can be accurately represented with piecewiseconstant functions and sharp edges would improve the characterization of the object's shape and size.

The unconstrained formulation of the TV image reconstruction problem is given by

$$
\min _{\Delta \epsilon} \frac{1}{2}\|J \Delta \epsilon-\Delta C\|_{2}^{2}+\alpha\|\nabla \Delta \epsilon\|_{1},
$$

where $\|\nabla \Delta \epsilon\|_{1}$ is the TV functional, i.e. the L1-norm of the gradient of the reconstructed image, and $\alpha$ is a regularization parameter that can be selected using the L-curve or similar method [17].

The TV functional is an optimal choice for many applications; however, it suffers from non-differentiability and a loss of contrast [30]. A common approach is to use a smooth approximation of the TV functional [29] [31], but this can compromise effective regularization or slow down convergence. An alternative that has been proposed to solve these issues is the use of the split Bregman formulation [15], which is an efficient formulation to solve L1-regularization approaches. Using this formulation, one aims at solving the constrained formulation of the TV image reconstruction problem

$$
\min _{\Delta \epsilon}\|\nabla \Delta \epsilon\|_{1} \quad \text { st. } \quad \frac{1}{2}\|J \Delta \epsilon-\Delta C\|_{2}^{2} \leq \sigma^{2}
$$

where $\sigma^{2}$ accounts for noisy data. The constrained formulation 6 enjoys further benefits with respect to the unconstrained formulation 7: it does not need to estimate the regularization parameter using the L-curve and the data constraint is imposed iteratively, which has the potential to achieve better reconstruction.

In the Split Bregman formulation constraints are imposed iteratively using the Bregman iteration as explained below.
In addition, introducing auxiliary variables allows separating L2- and L1-norm functionals in such a way that they can be solved analytically in two alternating steps, avoiding nondifferentiability issues that appear in standard approaches. To allow for splitting, we include new variables, $d_{x}, d_{y}, d_{z}$, and formulate a new problem equivalent to equation 7 , as follows:

$$
\begin{aligned}
& \min _{\Delta \epsilon, d_{x}, d_{y}, d_{z}}\left\|\left(d_{x}, d_{y}\right)\right\|_{1}+\left\|d_{z}\right\|_{1} \quad \text { st. } \\
& \quad \frac{1}{2}\|J \Delta \epsilon-\Delta C\|_{2}^{2} \leq \sigma^{2}, d_{x}=\nabla_{x} \Delta \epsilon, d_{y}=\nabla_{y} \Delta \epsilon, d_{z}=\nabla_{z} \Delta \epsilon(8)
\end{aligned}
$$

Using the Bregman iteration, the constrained problem 8 can be easily handled by using an equivalent unconstrained formulation for which constraints are imposed by adding a Bregman iteration $b_{i}$ for each constraint. Thus, equation 8 becomes

$$
\begin{array}{r}
\min _{\Delta \epsilon, d_{x}, d_{y}, d_{z}}\left\|\left(d_{x}, d_{y}\right)\right\|_{1}+\left\|d_{z}\right\|_{1}+\frac{\mu}{2}\left\|J \Delta \epsilon-\Delta C^{k}\right\|_{2}^{2} \\
+\frac{\lambda}{2}\left\|d_{x}-\nabla_{x} \Delta \epsilon-b_{x}^{k}\right\|_{2}^{2} \\
+\frac{\lambda}{2}\left\|d_{y}-\nabla_{y} \Delta \epsilon-b_{y}^{k}\right\|_{2}^{2} \\
+\frac{\lambda}{2}\left\|d_{z}-\nabla_{z} \Delta \epsilon-b_{z}^{k}\right\|_{2}^{2}
\end{array}
$$

where $k$ is the iteration number and the Bregman iterations are given by

$$
\begin{aligned}
b_{x}^{k+1} & =b_{x}^{k}+\nabla_{x} \Delta \epsilon^{k+1}-d_{x}^{k+1} \\
b_{y}^{k+1} & =b_{y}^{k}+\nabla_{y} \Delta \epsilon^{k+1}-d_{y}^{k+1} \\
b_{z}^{k+1} & =b_{z}^{k}+\nabla_{z} \Delta \epsilon^{k+1}-d_{z}^{k+1} \\
\Delta C^{k+1} & =\Delta C^{k}+\Delta C-J \Delta \epsilon^{k+1}
\end{aligned}
$$

The Bregman iterations impose constraints iteratively by adding the error back into the respective functionals [32] [15], thus making the solution of the problem 9 to converge to the solution of the constrained problem 8 . The last line in equation 10 enforces the data constraint, producing a sequence of solutions such that the solution error norm and the data fidelity term decrease monotonically.

To solve 9 we note that reconstructed image and auxiliary variables are independent of each other, so equation 9 can be split into several problems, one for each variable, and be solved sequentially. The permittivity change is solved analytically by solving the following linear system:

$$
\begin{gathered}
\left(\mu J^{T} J+\lambda \sum_{i=x, y, z} \nabla_{i}^{T} \nabla_{i}+\gamma I\right) \Delta \epsilon= \\
\mu \Delta C^{k}+\lambda \sum_{i=x, y, z} \nabla_{i}^{T}\left(d_{i}-b_{i}\right)+\gamma \Delta \epsilon^{k}
\end{gathered}
$$

where the last term on both the LHS and RHS is added for stability. Auxiliary variables are given by the following 

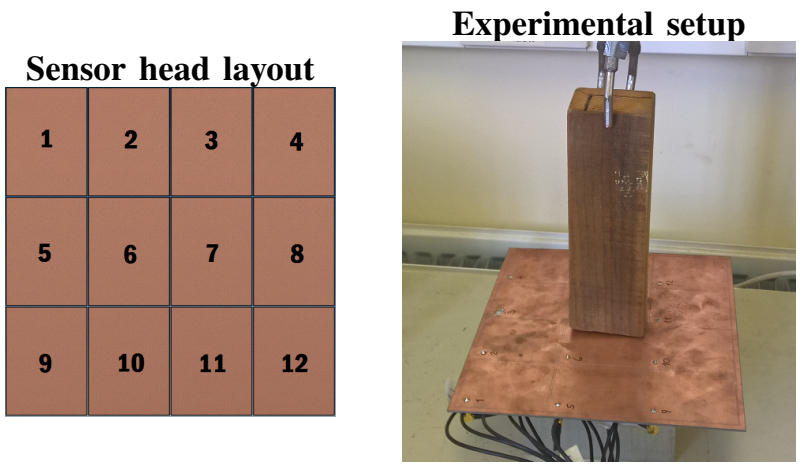

Fig. 1: Sensor head layout and experimental setup for training data (sample 1).

shrinkage formulae [Goldstein 2009]:

$$
\begin{aligned}
d_{x}^{k+1} & =\max \left(s^{k}-1 / \lambda, 0\right) \frac{\nabla_{x} \Delta \epsilon^{k+1}+b_{x}^{k}}{s^{k}} \\
d_{y}^{k+1} & =\max \left(s^{k}-1 / \lambda, 0\right) \frac{\nabla_{y} \Delta \epsilon^{k+1}+b_{y}^{k}}{s^{k}} \\
s^{k} & =\sqrt{\sum_{i=x, y, z}\left|\nabla_{i} \Delta \epsilon^{k+1}+b_{i}^{k}\right|^{2}} \\
d_{z}^{k+1} & =\max \left(\left|\nabla_{z} \Delta \epsilon^{k+1}+b_{z}^{k}\right|-1 / \lambda, 0\right) \frac{\nabla_{z} \Delta \epsilon^{k+1}+b_{z}^{k}}{\left|\nabla_{z} \Delta \epsilon^{k+1}+b_{z}^{k}\right|}
\end{aligned}
$$

\section{EXPERIMENTAL PROCEDURE}

The TV algorithm parameters, $P_{T V}$, that are going to be optimised in order to produce the best result are $\mu, \lambda$ and $\gamma$. The algorithm also has a Bregman iteration count, $n_{b}$, which controls how many iterations are used in the reconstruction. The higher this is, the more the data constraint is imposed. Decreasing $\mu$ (weight of the data fidelity term) and increasing lambda (weight of the gradient constraints) leads to lower $n_{b}$, requiring fewer iterations to converge. Optimal selection of these parameters can reduce computational time significantly [19]. Tikhonov, equation 5, also requires input parameters, $P_{\text {tikhonov }}$, but only 2, and these are $\alpha_{1}$ and $\alpha_{2}$.

ECT is greatly affected by the distance of the object to the sensor [4] which means that objects imaged with ECT will have a much more accurately reconstructed front surface (the closest surface to the ECT sensor) than the other surfaces further away. For this reason, the closest surface is used to localise the object. This surface is referred to as the front surface. The other sides of the object are likely to be reconstructed with less accuracy.

The software used in these experiments is DeTECT [7], a MATLAB based toolbox used for ECT simulation, reconstruction and visualisation. It is specifically designed for use with planar array ECT systems. It uses a finite difference method (FDM) for the forward problem modelling.

A test in this paper is defined as reconstructing a 3D permittivity distribution for a particular set of parameters and detecting the distance of the front surface of the reconstructed object.

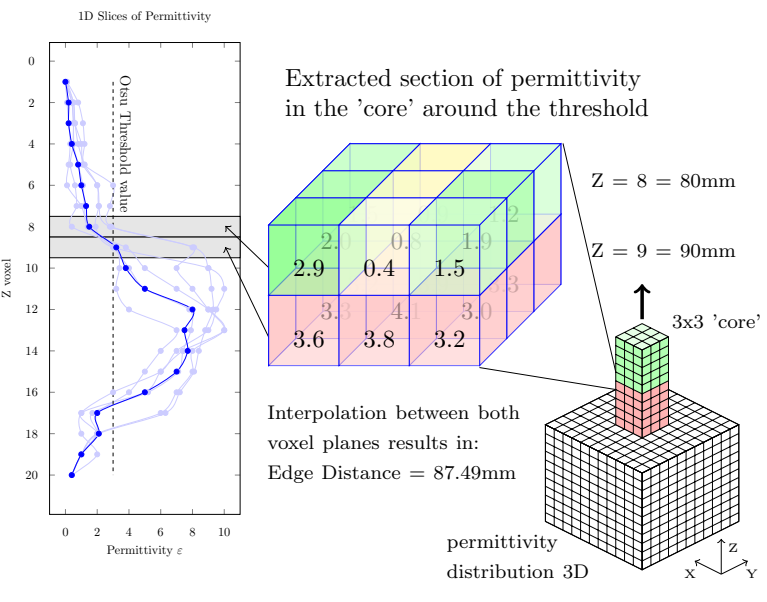

Fig. 2: Distance detection method showing interpolation between voxel levels around the Otsu Threshold value. GREEN - Smaller than threshold, RED - Bigger than threshold.

The detected distance, $d_{d}$, is then compared to the expected distance, $d_{e}$, and used to compute the difference, $d_{d i f f}$. A 2D slice is also taken from the original permittivity distribution and an inclusion is extracted from it through segmentation. This inclusion is then compared to the expected inclusion and a score is calculated to show how accurate it is. This results in 'distance difference' and 'reconstruction score' qualifiers for all tests.

All tests will use both simulated capacitance data as well as real captured capacitance data and the same Jacobian to reconstruct the permittivity. The simulated capacitance data is obtained by simulating a block with DeTECT , suspended at $3 \mathrm{~cm}$ incrementing heights above the sensor head which is a $3 \times 4,12$ electrode sensor as shown in figure 1 with external dimensions of $20 \mathrm{~cm}$ by $20 \mathrm{~cm}$. The real captured capacitance data is measured with the same distances using a wooden block as the inclusion. The Jacobian is computed with DeTECT at a resolution of $21 \times 21 \times 21$ in the $x, y$ and $z$ directions respectively and then used with the capacitance data to reconstruct the 3D permittivity distribution. The sensor used is the same as that referred to as 'original sensor' in previous work on planar array ECT [7].

\section{A. Front Surface Distance Detection (FSDD)}

The distance $d_{d}$ to the front surface is calculated in several stages. As the reconstruction is not perfect, the front surface may not be smooth which means that if the distance was measured from a single point on the surface it might not be representative of the actual distance of object. For this reason the distance detection is performed on multiple points and an average distance detected is taken from this. The algorithm 1 explains how the distance is detected and a visualisation of this can be seen in figure 2 . 

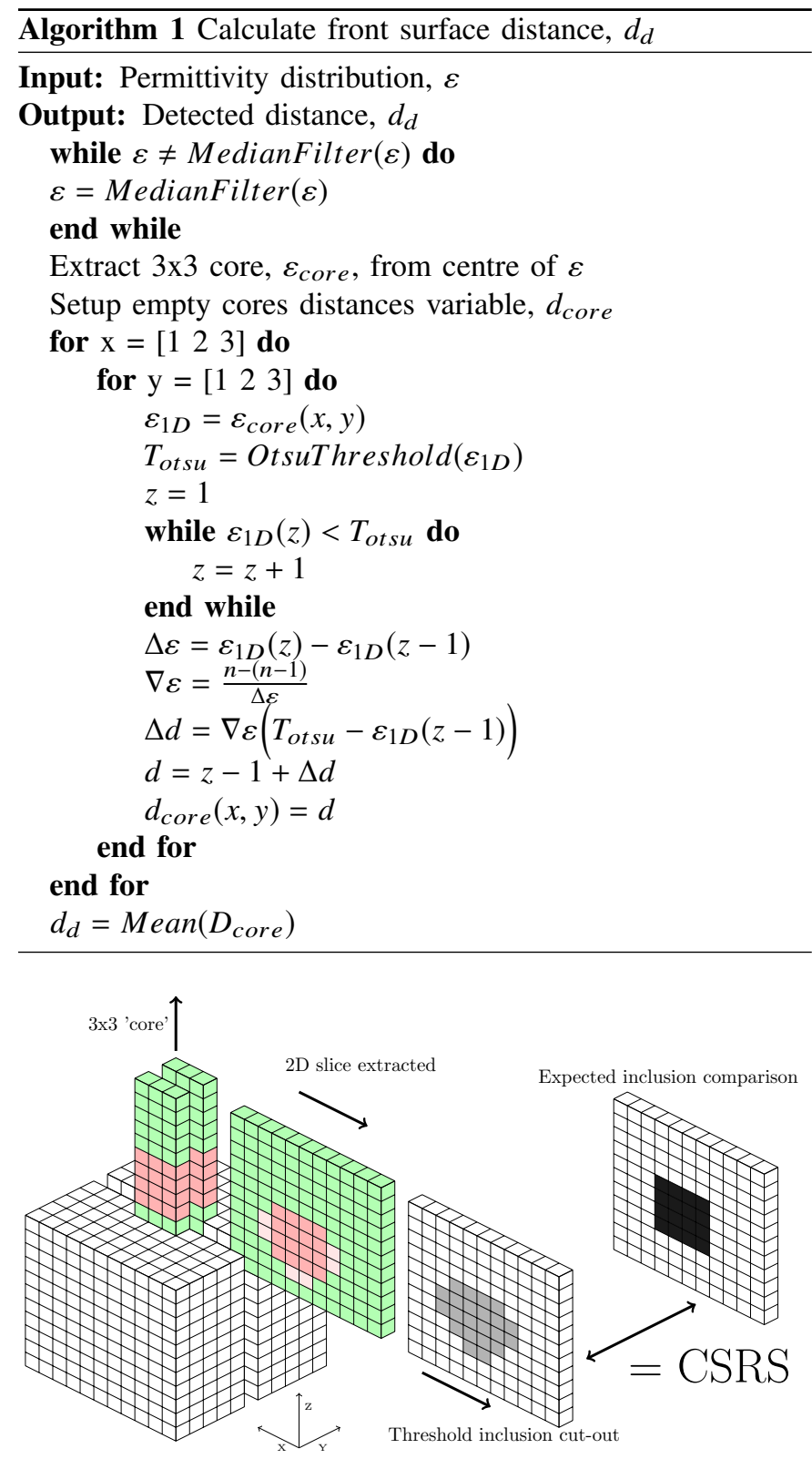

Fig. 3: 2D slice taken from centre of 'core' and then the inclusion cut-out based on threshold values from the FSDD method.

\section{B. Cross-section Reconstruction Score (CSRS)}

The distance $d_{d}$ from FSDD is only useful if the inclusion that was reconstructed is close in shape to that of the actual inclusion otherwise the detected distance could be coincidental and not representative of a good parameter set. For this reason every test also checks a cross-section of the permittivity distribution to check on the shape of the reconstructed inclusion. Algorithm 2 explains how this score is calculated.

\section{Parameter Optimisation}

Parameter optimisation is a hard problem. Several methods have been proposed to optimise the regularisation parameter

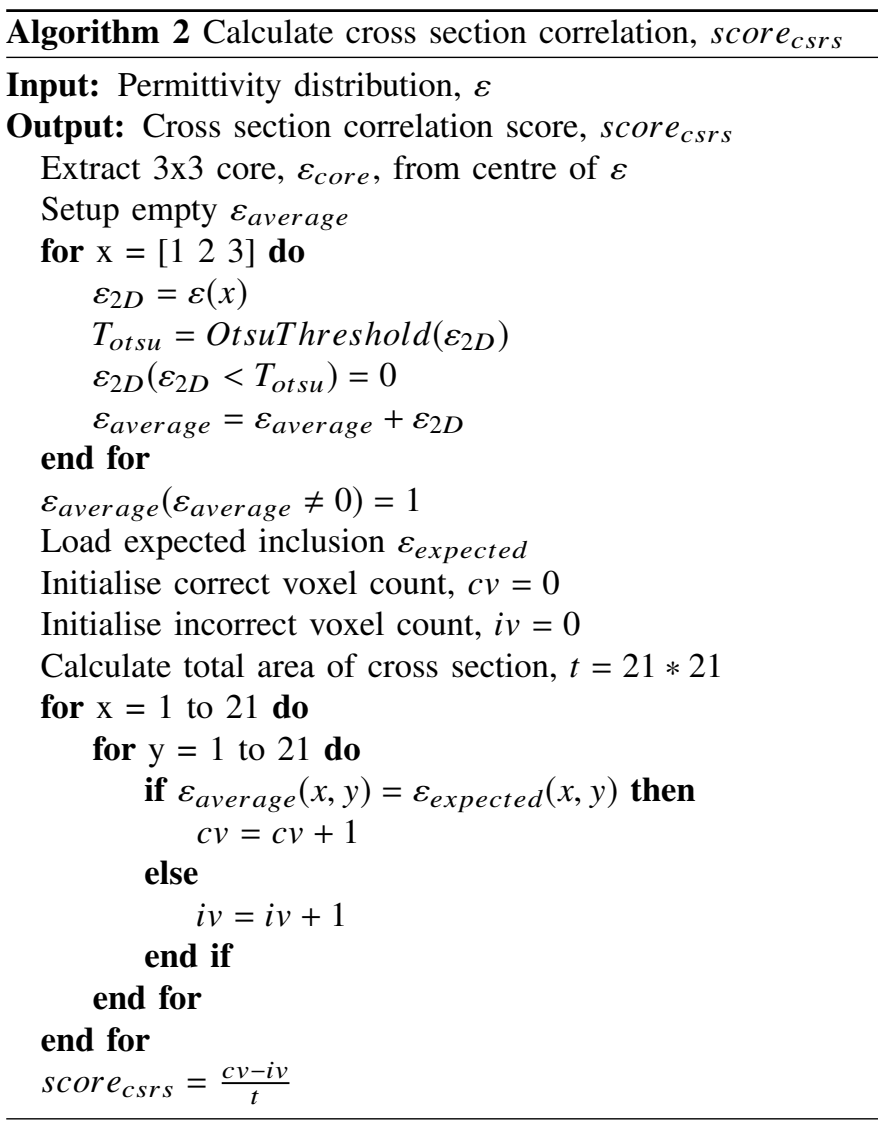

for Tikhonov [29]. Performance of these methods depends on the application and data dependent, so methods are generally compared for a new application [18]. Automatic selection of other hyperparameters, such as those in the split Bregman formulation (they are not regularization parameters), is still an open issue, where some guidelines have been previously proposed [15] [19] [33]. For optimising TV and Tikhonov parameters three methods were tried.

1) Brute Force: A 'brute force' method of evenly searching all combinations of parameters in a given size range was attempted. It was found however that if the number of parameter combinations was too large then the optimisation took too long and if too small then the gaps between parameter values was too large.

2) Neighbour Search: This method used a starting set of parameters and then varied them to try improving the results. If the reconstruction scored badly, the parameters step size was increased and then decreased as the reconstruction score improved thus aiming to converge on an optimal solution. The starting parameter set used was that of the optimal parameter set of the 'neighbouring' reconstruction distance. This method produced good results but not consistently enough.

3) Genetic Algorithm Optimisation (GAO): The GA was implemented using the MATLAB Optimization Toolbox [34]. It uses a multi-start global search method which means that it has many start points and searches for the best parameter set regardless of parameter values. It is attempting to find the global minima of difference 


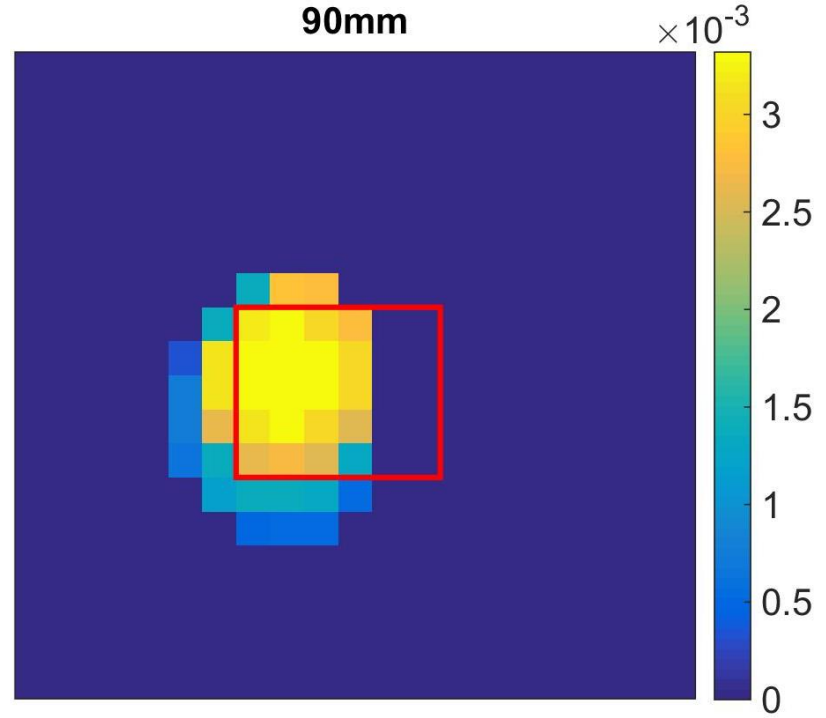

Fig. 4: TV reconstruction of real data captured with object at $90 \mathrm{~mm}$ from sensor head. Red box shows expected location of object.

between FSDD and expected distance by searching for local minima and selecting the best one. The GA is given 2 stopping criteria to prevent it over-searching. It is given a maximum number of local minima, $L M_{\max }$, found before it then assigns the lowest one as the solution. It also stops if it finds a local minima lower than a chosen threshold, $T_{\text {diff }}$, as this is deemed a suitable solution. However if a solution has a CSRS lower than the threshold, $T_{c s r s}$, then this solution is not counted as a valid solution.

\section{EXPERIMENTAL RESULTS}

For training of reconstruction parameters, real capacitance data was collected at $30 \mathrm{~mm}, 60 \mathrm{~mm}, 90 \mathrm{~mm}$ and $120 \mathrm{~mm}$ as described in the experimental procedure. Simulated data was then created for the same distances. These two sets are referred to as Sample 1.

For both Sample 1 data, GAO was used to find optimal parameters at each distance, $d_{e}$, of the wooden block. GAO used the settings, $T_{\text {diff }}=0.05 d_{e}, T_{c s r s}=50$ and $L M_{\max }=10$. These values were selected as they gave the best balance between testing time and testing accuracy.

The TV algorithm used a $n_{b}$ value of 10 to allow for a balance between quick reconstruction $(\approx 2 s)$ and reconstruction accuracy. Given the relation between $\mu$ and $n_{b}$ [19], one can fix one of these parameters and modify the other one.

Figure 4 shows a reconstruction by the TV algorithm of an inclusion at $90 \mathrm{~mm}$ from the sensor head. In this reconstruction and all subsequent reconstructions shown the sensor is located at the top of the image as the images are mimicking a landmine detection scenario where the viewing direction would be down through the ground. The red square in the image shows the expected location of the inclusion.

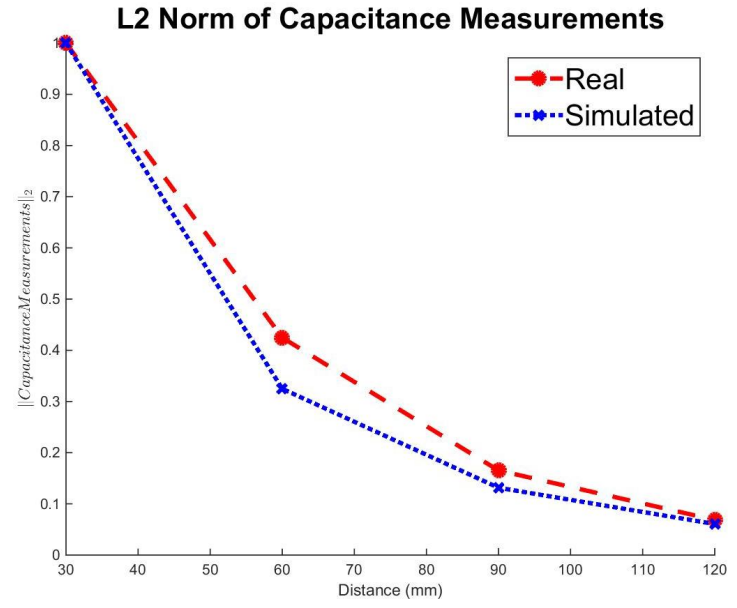

Fig. 5: Capacitance-Distance relationship curve.

\section{A. Capacitance Measurements}

The raw capacitance measurements of Sample 1 can be seen to show an interesting relationship between capacitance and distance of object. As the distance of the object increased from the sensor head, the euclidean norm of the raw capacitance measurements proportionally changed too. This can be seen in figure 5.

This Capacitance-Distance relationship allows an 'estimated' distance to be found by simply taking a capacitance measurement, before any reconstruction is performed. Using this estimated distance, appropriate parameters can be chosen by using the Distance-Parameter relation found through Parameter Optimisation.

This relationship is very specific to this experimental setup, a higher permittivity object observed at different distances in a lower permittivity surrounding. However for other situations the relationship can be found through experimental calibration in that environment which will give a unique CapacitanceDistance relationship.

\section{B. Parameter Optimisation Comparison}

The GAO method found 'optimal' parameters for both TV and Tikhonov reconstructions for each distance tested. This data now forms Distance-Parameter relationship curves which can be used for Automatic Parameter Selection.

A brief look at the individual parameters found by the optimisation showed that the TV parameters were a lot less affected by their individual magnitudes and more affected by the ratio to each other. For example using parameters of 1,1 and 1 for $\mu, \gamma$ and $\lambda$ respectively was almost identical to using $1 e^{-10}, 1 e^{-10}$ and $1 e^{-10}$ respectively. However for Tikhonov the parameters magnitude was far more important as they effectively act as weights to decide how much regularisation is applied. Therefore they are not as strongly linked to each other but more to their own values. 


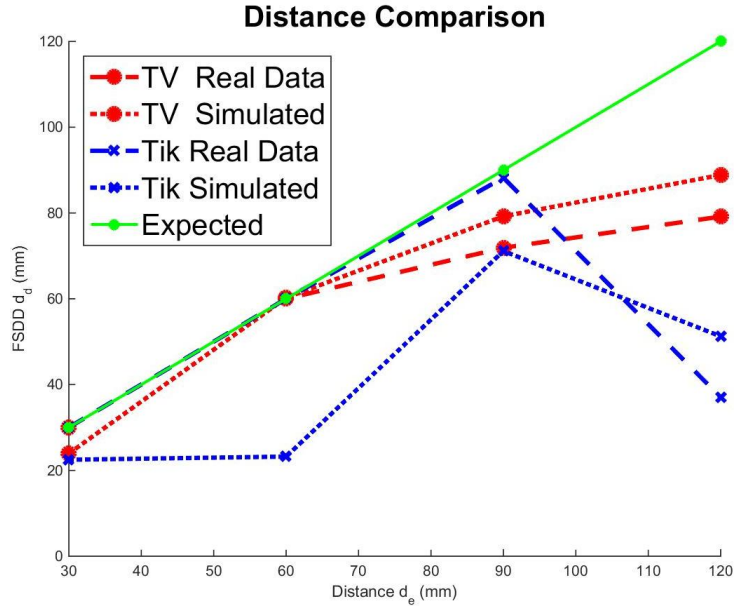

Fig. 6: Distance detected from Sample 1 reconstructions using optimal parameters.

\section{Distance Detection Comparison}

The TV algorithm was much more consistent when detecting distance than Tikhonov which can be seen in figure 6. The TV reconstructions of both real and simulated Sample 1 data were not accurate beyond $60 \mathrm{~mm}$ but the error was consistent. If the distance detection error is consistent it means that a scaling factor could be used to get more accurate detection measurements. In these results when the inclusion was at $120 \mathrm{~mm}$ the detected distance was $\approx 80 \mathrm{~mm}$. Therefore in practice a detected $80 \mathrm{~mm}$ reconstruction could be scaled to give a $120 \mathrm{~mm}$ reading as an actual $80 \mathrm{~mm}$ inclusion would result in $\mathrm{a} \approx 70 \mathrm{~mm}$ reading as shown by figure 6 .

The Tikhonov results were far more inconsistent. It performed poorly at $120 \mathrm{~mm}$ and was not consistent with results at $90 \mathrm{~mm}$, therefore the same scaling theory used for TV would not work.

Both algorithms performed extremely well at $30 \mathrm{~mm}$ and $60 \mathrm{~mm}$ when optimised with real data but with simulated data the results are not so good.

Overall it shows that both TV and Tikhonov show an ability to detect up to $90 \mathrm{~mm}$ with limited error and promise of being able to potentially detect up to $120 \mathrm{~mm}$. This not only proves the ability of the algorithms and DeTECT software but also the hardware used to collect the data [7].

\section{Automatic Parameter Selection}

Automatic Parameter Selection (APS) uses the ParameterDistance relationship curves for both TV and Tikhonov combined with the Capacitance-Distance relationship curve, to automatically select the optimal parameter set for a given capacitance reading. Algorithm 3 describes how this is performed.

In order to assess the ability of algorithm 3, new capacitance data sets, Sample 2, were collected with the same setup as before, but with the inclusions at varying distances and with
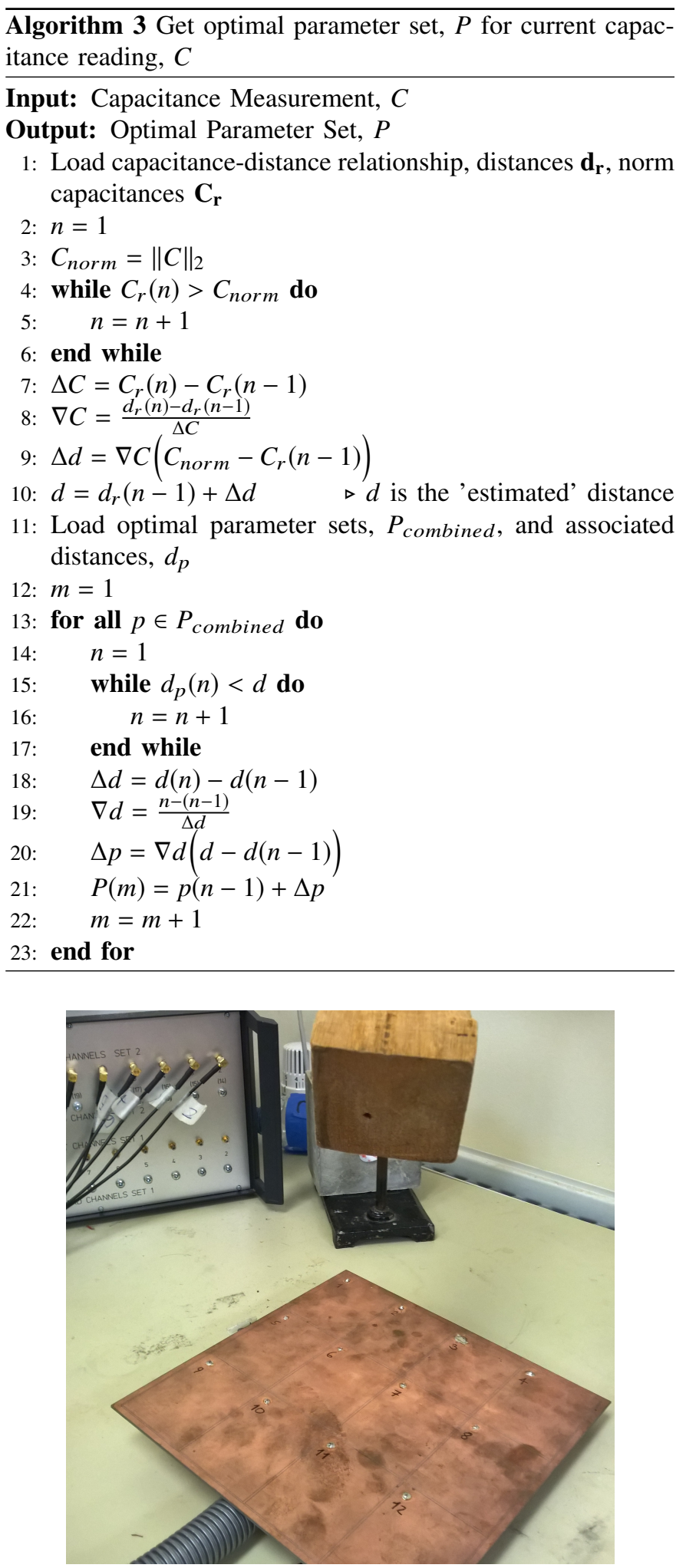

Fig. 7: Experimental setup of Sample 2 gathered for testing of Automatic Parameter Selection.

a different object. Sample 2 only consists of real data. The experimental setup can be seen in figure 7 . The object was randomly orientated to produce a very different scenario to the real data in Sample 1. The distances of the object are also random so that they lie in between the distances of the 

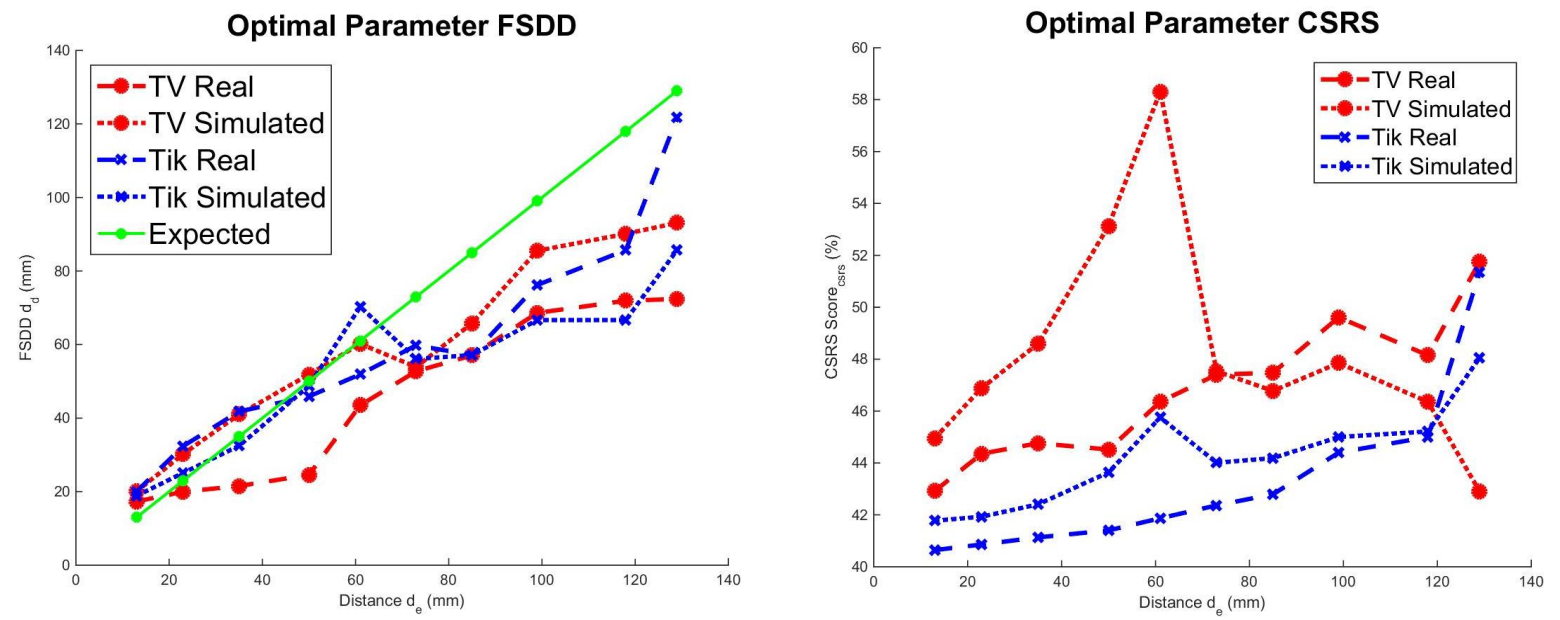

Fig. 8: Detected distances $d_{d}$ against the expected distance $d_{e}$ for TV and Tikhonov reconstructions on Sample 2 data.

training data. For this reason interpolated parameters are used instead of exact optimal parameters. Using the exact optimal parameters found through training on Sample 1 would be expected to get the same results. If interpolated parameters from algorithm 3 were used, then this should result in a parameter set which is yet untested, and if this is able to reconstruct the inclusion accurately, then this helps to support a relationship between parameters and distance.

Figure 8 shows the detected distances, $d_{d}$, using parameter sets from algorithm 3 as well as the CSRSs of these reconstructions. Looking at detected distance, $d_{d}$ shows that all the optimal parameters sets perform very similarly. There appears to be 2 distinct regions on the graph where the behaviour differs. The first region is up to $60 \mathrm{~mm}$ which is roughly the typical burial depths of landmines [35]. The reconstruction algorithms behave similarly and are on average accurate to within $7.2 \mathrm{~mm}$. Tikhonov reconstruction was able to detect objects with an average error of $5.7 \mathrm{~mm}$ between distances $30 \mathrm{~mm}-60 \mathrm{~mm}$ and TV reconstruction had an average error of $8.7 \mathrm{~mm}$. Above $60 \mathrm{~mm}$ is quite deep for a landmine and here there seems to be an constant shift in detected distance from expected distance. If this is consistent in future experiments it could mean that the values could be scaled such that for example a detected distance of $100 \mathrm{~mm}$ actually represents an object at $120 \mathrm{~mm}$. The percentage error from expected distance to detected distance is quite consistent at $\approx 30 \%$.

Using APS both algorithms are still able to produce similar results to the Sample 1 results 6, and Tikhonov is even able to reconstruct up to $130 \mathrm{~mm}$ with only $\approx 4 \%$ error. It could be that the new data sets, in Sample 2, are better quality with less noise and fewer background signals. But the consistency of the distance detection for both reconstruction methods and optimisation parameter sets shows that for this application there is some relationship between parameter values and front surface distance of an object.

Both TV and Tikhonov have similar FSDDs and therefore it could be a one-off result meaning that Tikhonov might perform better in some tests and TV in other tests. However when comparing CSRSs, TV performs much better on average. In figure 8 the TV CSRSs are far better at almost all distances. This means that TV is able to reconstruct the inclusions with a much better overall shape, not just the front surface and therefore the TV reconstructions are more reliable as the inclusion can be located with more certainty.

Figure 9 shows reconstructions of Sample 2 data using optimal parameters trained from real data. The red lines indicate the expected location of the top surface. Both reconstruction methods produce similar images and none of them perfectly find the top surface.

The TV reconstructions have slightly better defined edges than the Tikhonov reconstructions. This is to be expected due to the nature of the TV algorithm. It means that the overall object edges can be defined with more certainty whereas with Tikhonov the exact location of the inclusion boundary is estimated using Otsu thresholding select edges from the blurred inclusion.

Landmine detection is far safer if objects can be accurately reconstructed and located. The TV reconstructions show the inclusion location more accurately and the shape can be assumed with more certainty than Tikhonov reconstructions.

The aim of automatic parameter selection was not to produce the best images but to remove the need for an expert to select the reconstruction parameters. The images produced show that it is possible to select parameters automatically. Through some manual parameter selection during experimentation is was observed that changing the parameters can drastically change the appearance of the reconstructed image. Thus, automatic parameter selection can lead to more consistent distance detection.

\section{CONCLUSION}

In order to use ECT for landmine detection, distance detection of planar array ECT needs to be consistent and reliable. Planar array ECT suffers from poor reconstruction of inclusions 


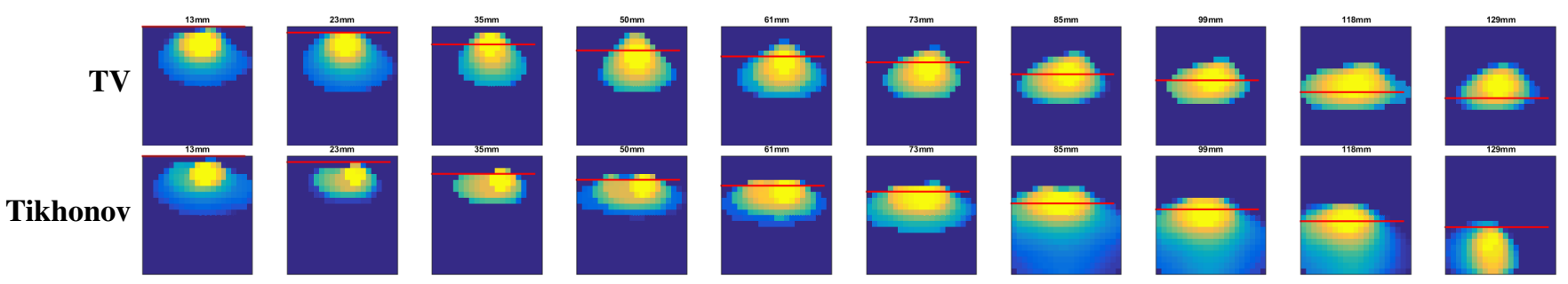

Fig. 9: Detected distances $d_{d}$ against the expected distance $d_{e}$ for TV and Tikhonov reconstructions on Sample 2 data.

further away from the sensor. A way of improving this is through optimisation of the image reconstruction parameters. However selecting appropriate parameters is often done by

The development of the APS method has removed the need for expert calibration of image reconstruction parameters for TV and Tikhonov. The initial training of the APS method showed that there was not much difference between training with simulated data or real data of Sample 1 and both reconstruction algorithms performed similarly. However when Sample 2 data was used to test the automatic parameter selection algorithm Tikhonov was more consistent in distance detection than TV although TV was still producing good results at smaller distances. These results could be explained by the fact that selecting Tikhonov parameters is more robust due to the noniterative nature and fewer parameters of the algorithm whereas TV relies more on iteration count and the larger number of parameters. It could be that better TV optimal parameters can be found using different training methods instead the GA used in this work.

The main conclusion of this paper is the ability to automatically select parameters and produce results which are consistent and quick without any expert calibration. Also for future comparisons to be made with this work, the clear explanation of the FSDD and CSRS scoring has created quantifiers for accurate distance scoring whilst making sure the reconstruction is showing expected inclusion shape.

\section{ACKNOWLEDGEMENT}

This work is sponsored by Find A Better Way, a landmine detection and clearance charity and the University of Bath Alumni Fund. JFPJ Abascal has received funding from the European Union's Horizon 2020 research and innovation programme under the Marie Sklodowska-Curie grant agreement No 701915.

\section{REFERENCES}

[1] S. Huang, A. Plaskowski, C. Xie, and M. Beck, "Capacitance-based tomographic flow imaging system," Electronics Letters, vol. 24, no. 7, p. 418, mar 1988 .

[2] S. M. Huang, A. B. Plaskowski, C. G. Xie, and M. S. Beck, "Tomographic imaging of two-component flow using capacitance sensors," Journal of Physics E: Scientific Instruments, vol. 22, no. 3, pp. 173-177, 1989.
[3] A. V. Mamishev, K. Sundara-Rajan, F. Yang, Y. Du, and M. Zahn, "Interdigital sensors and transducers," Proceedings of the IEEE, vol. 92, no. 5, pp. 808-844, may 2004.

[4] X. Hu and W. Yang, "Planar capacitive sensors âĂŞ designs and applications," Sensor Review, vol. 30, no. 1, pp. 24-39, 2010.

[5] Z. Ye, R. Banasiak, and M. Soleimani, "Planar array 3D electrical capacitance tomography," Insight: Non-Destructive Testing and Condition Monitoring, vol. 55, no. 12, pp. 675-680, dec 2013.

[6] ICBL-CMC. (2015) Landmine Monitor.

[7] C. Tholin-Chittenden and M. Soleimani, "Planar Array Capacitive Imaging Sensor Design Optimization," IEEE Sensors Journal, vol. 17, no. 24, pp. 8059-8071, dec 2017.

[8] Y. Xiao and J. Yang, "A Fast Algorithm for Total Variation Image Reconstruction from Random Projections," jan 2010.

[9] Y. Wang, J. Yang, W. Yin, and Y. Zhang, "A New Alternating Minimization Algorithm for Total Variation Image Reconstruction," SIAM Journal on Imaging Sciences, vol. 1, no. 3, pp. 248-272, jan 2008.

[10] B. Li and D. S. Que, "Medical images denoising based on total variation algorithm," Procedia Environmental Sciences, vol. 8, pp. 227-234, jan 2011.

[11] M. Lohvithee, A. Biguri, and M. Soleimani, "Parameter selection in limited data cone-beam CT reconstruction using edge-preserving total variation algorithms," Physics in Medicine \& Biology, vol. 62, no. 24, pp. 9295-9321, nov 2017.

[12] E. Al Hosani, M. Zhang, J. F. P. J. Abascal, and M. Soleimani, "Imaging metallic samples using electrical capacitance tomography: forward modelling and reconstruction algorithms," Measurement Science and Technology, vol. 27, no. 11, p. 115402, nov 2016.

[13] J. Chen, M. Zhang, Y. Liu, J. Chen, and Y. Li, "Image reconstruction algorithms for electrical capacitance tomography based on ROF model using new numerical techniques," Measurement Science and Technology, vol. 28, no. 3, p. 035404, mar 2017.

[14] P. L. Combettes and J. C. Pesquet, "Proximal splitting methods in signal processing," Springer Optimization and Its Applications, vol. 49, pp. $185-212,2011$.

[15] T. Goldstein and S. Osher, "The Split Bregman Method for L1Regularized Problems," SIAM Journal on Imaging Sciences, vol. 2, no. 2, pp. 323-343, 2009.

[16] S. Setzer, "Split bregman algorithm, douglas-rachford splitting and frame shrinkage," in Lecture Notes in Computer Science (including subseries Lecture Notes in Artificial Intelligence and Lecture Notes in Bioinformatics). Springer, Berlin, Heidelberg, 2009, vol. 5567 LNCS, pp. $464-476$.

[17] P. C. Hansen and D. P. O'Leary, "The Use of the L-Curve in the Regularization of Discrete Ill-Posed Problems," SIAM Journal on Scientific Computing, vol. 14, no. 6, pp. 1487-1503, nov 1993.

[18] J.-F. P. J. Abascal, S. R. Arridge, R. H. Bayford, and D. S. Holder, "Comparison of methods for optimal choice of the regularization parameter for linear electrical impedance tomography of brain function," Physiological Measurement, vol. 29, no. 11, pp. 1319-1334, nov 2008. 
[19] J. F. P.-J. Abascal, J. Chamorro-Servent, J. Aguirre, S. Arridge, T. Correia, J. Ripoll, J. J. Vaquero, and M. Desco, "Fluorescence diffuse optical tomography using the split Bregman method." Medical physics, vol. 38, no. 11, pp. 6275-84, 2011.

[20] J. Abascal, P. Montesinos, E. Marinetto, J. Pascau, and M. Desco, "Comparison of total variation with a motion estimation based compressed sensing approach for self-gated cardiac cine MRI in small animal studies." PloS one, vol. 9, no. 10, p. e110594, oct 2014.

[21] C. He, C. Hu, W. Zhang, B. Shi, and X. Hu, "Fast total-variation image deconvolution with adaptive parameter estimation via split Bregman method," Mathematical Problems in Engineering, vol. 2014, pp. 1-9, feb 2014.

[22] Y. Xu, Y. Pei, and F. Dong, "An adaptive Tikhonov regularization parameter choice method for electrical resistance tomography," Flow Measurement and Instrumentation, vol. 50, pp. 1-12, aug 2016.

[23] P. Hansen, "The L-curve and its use in the numerical treatment of inverse problems," IN COMPUTATIONAL INVERSE PROBLEMS IN ELECTROCARDIOLOGY, ED. P. JOHNSTON, ADVANCES IN COMPUTATIONAL BIOENGINEERING, vol. 4, pp. 1-24, 1999.

[24] M. Cheney, D. Isaacson, J. C. Newell, S. Simske, and J. Goble, "NOSER: An algorithm for solving the inverse conductivity problem," International Journal of Imaging Systems and Technology, vol. 2, no. 2, pp. 66-75, 1990.

[25] C. Wu, J. He, and X. Zou, "A Genetic Algorithm Approach for Selecting Tikhonov," in 2008 IEEE Congress on Evolutionary Computation (IEEE World Congress on Computational Intelligence). IEEE, jun 2008, pp. 3980-3983.

[26] Y. Serinagaoglu Dogrusoz and A. Mazloumi Gavgani, "Genetic algorithm-based regularization parameter estimation for the inverse electrocardiography problem using multiple constraints," Medical and Biological Engineering and Computing, vol. 51, no. 4, pp. 367-375, 2013.

[27] M. Zhang, L. Ma, Z. Ye, C. L. Yang, H. Y. Wei, R. Banasiak, and M. Soleimani, "Near subsurface 3D imaging using planar array : EIT, ECT, MIT," in 7th World Congress on Industrial Process Tomography, WCIPT7, no. September. Krakow, Poland: ISIPT, 2013, pp. 2-5.

[28] A. Neubauer, "Tikhonov regularisation for non-linear ill-posed problems: Optimal convergence rates and finite-dimensional approximation," Inverse Problems, vol. 5, no. 4, pp. 541-557, 1989.

[29] C. Vogel, Computational methods for inverse problems. Society for Industrial and Applied Mathematics, jan 2002, vol. 23.

[30] M. Burger and S. Osher, "A guide to the TV zoo," in Lecture Notes in Mathematics, 2013, vol. 2090, pp. 1-70.

[31] L. I. Rudin, S. Osher, and E. Fatemi, "Nonlinear total variation noise removal algorithm," Physica D: Nonlinear Phenomena, vol. 60, no. 1-4, pp. 259-268, 1992.

[32] S. Osher, M. Burger, D. Goldfarb, J. Xu, and W. Yin, "An Iterative Regularization Method for Total Variation-Based Image Restoration," Multiscale Modeling \& Simulation, vol. 4, no. 2, pp. 460-489, 2005.

[33] J. F. Abascal, M. Abella, E. Marinetto, J. Pascau, and M. Desco, "A novel prior- and motion-based compressed sensing method for smallanimal respiratory gated CT," PLOS ONE, vol. 11, no. 3, p. e0149841, mar 2016.

[34] T. Coleman, M. A. Branch, and A. Grace, "Matlab Optimization Toolbox," p. 42, 2008.

[35] R. Keeley, "Understanding landmines and mine action," no. September, pp. 2-7, sep 2003. 